\title{
Stroboscopic Laser Diagnostics for Detection of Ordering in One-Dimensional Ion beam.
}

\author{
R. Calabrese†, V. Guidi†, P.Lenisa†, E. Mariotti§, L. Moi§ and U.Tambini† \\ $\dagger$ †ipartimento di Fisica dell'Università, I-44100 Ferrara, Italy; INFN - Sezione di Ferrara, Italy. \\ $\S$ Dipartimento di Fisica dell'Università, I-53100 Siena, Italy.
}

(August 29, 2019)

\begin{abstract}
A novel diagnostic method for detecting ordering in one-dimensional ion beams is presented. The ions are excited by a pulsed laser at two different positions along the beam and fluorescence is observed by a group of four photomultipliers. Correlation in fluorescence signals is firm indication that the ion beam has an ordered structure.
\end{abstract}

32.80.-t, 41.75.-i, 29.20

Typeset using REVTEX 


\section{INTRODUCTION}

In the past few years the interest in the field of ion crystallization has become increasingly stronger from both theoretical and experimental points of view. This research effort has led to the successful detection of crystallization in an ion trap [1] and in a mini-quadrupole storage ring [2]. In these systems, ions are at rest in the laboratory frame and the transition to the ordered state is achieved by laser cooling [3].

At the same time, the possibility has been suggested to cool an ion beam in a storage ring to the extent that a crystalline phase is reached even in such system, in which particles travel at high velocity [4]. In this case, compaction of phase-space of the ion beam is obtained by electron [5] and laser cooling techniques. Recently, an ion storage ring dedicated to crystallization studies has been proposed [6].

Among the items one should consider in designing such a ring is the problem of cooling the ion beam and the diagnostics of the ordered state. It is known that an ion beam can be efficiently laser-cooled only longitudinally but there is no experimentally tested technique that allows cooling of the transverse degrees of freedom of the ion beam as efficiently as longitudinally. Although some proposals for an efficient transverse cooling are under consideration, the lack of such a technique is a severe obstacle to the reaching of any ordered state.

The diagnostics of the ordered state is another crucial point, because a clear and unambiguous detection of the crystalline state is necessary to validate experimental findings. For the case of ions in traps, one can use a CCD-based detection since ions are practically at rest [2].

Unfortunately, to our knowledge, no satisfactory method to detect ordering of an ion beam by resolving the single ion has been proposed so far. It has been suggested that Schottky-noise-based pick-ups might detect the passage of the single particle of the beam but, in a storage ring, the bandwidth of such an instrument would be greater than $100 \mathrm{GHz}$, that is by far higher than any available device can achieve. Among optical methods, some 
possibilities are described in Ref. [7]; these instruments should provide a response correlated with ion order but they would never allow a direct and unambiguous evidence of the position of the single ion.

In this paper we propose a fluorescence-based method to detect ordering in a onedimensional ion beam.

\section{BASIC PRINCIPLES OF THE METHOD}

A pulsed laser - resonant with the travelling ions - is split in two parts, which simultaneously cross the ion beam at right angle at two nearby positions along the storage ring (see Fig.1). This laser-to-ion crossing area is followed by four photomultipliers which detect the photons emitted by the ions that have previously been excited by the laser beams. The signals recorded by the photomultipliers are analyzed when one laser beam is moved with respect to the other one. In the absence of ordering, no correlation in fluorescence signals should be recorded while changing the relative distance of the two laser beams. On the contrary, if a string were obtained as a result of cooling, a strong correlation between the signals should be observed. Suppose that one of the four photomultipliers detects the fluorescence of an ion excited by one of the laser beams. If one of the other three photomultipliers detects a simultaneous fluorescence signal, it means that the other laser has interacted with another ion in the string, in turn indicating that the distance between the two ion-to-laser crossing points is an integer multiple of the string interparticle spacing. Then, by slightly moving the second laser beam, the correlation signal should vanish. A sort of periodical dependence on the distance between the laser beams should appear, whose contrast will mainly depend on the detection efficiency.

The diagnostics is conceived with the aim of detecting ordering within a string of ions in a storage ring. When an ion beam in a storage ring undergoes sufficiently strong cooling, a phase transition to an ordered state is expected to occur [8]. The simplest ordered structure is a one-dimensional string. For this system the degree of ordering increases as temper- 
ature decreases without any sharp transition as is the case for three-dimensional systems [9] [10]. Typical values of the interparticle spacing for the string configuration lie between $s=10-100 \mu \mathrm{m}$; for the following we shall assume $s=50 \mu \mathrm{m}$. At non-zero temperature, ions are expected to oscillate both in the transverse and longitudinal directions (incoherent motion). Moreover, for a string longitudinal oscillations of equilibrium positions can also occur (coherent motion) through long-range waves. For the diagnostic system concerned through this paper the distance between the two laser beams can be chosen to match interparticle spacing of the string. In this case, the effect of coherent motion is uneffective as the nearest-neighbor spacing is relatively uniform. The effect of long wavelength on interparticle oscillations is negligible for a relative distance of a few lattice steps. An analytical evaluation of this effect can be found in Ref. [11]. Short wavelengths mostly affect the fluctuations in interparticle spacing, $\delta s$. Based on Ref. [11], a rough estimate holds: $\left\langle\delta s^{2}\right\rangle / s^{2} \simeq 1 / \Gamma$, where $\Gamma$ is the plasma parameter for the ion beam. When the ion beam is being cooled $\delta s$ becomes even lower than $s$; as an example, at $\Gamma=100, \delta s=5 \mu \mathrm{m}$. If the distance between the laser beams were larger than one interparticle spacing, the correlation signal should become progressively weaker.

The configuration of the diagnostic device can also compensate for a possible influence of transverse oscillations. Since the laser beams cross the string orthogonally and their spots are small enough that they do not overlap, the ions suffering transverse oscillations in the direction of the laser can always be resonant, irrespective of their coordinates along that axis. Ion oscillations in direction orthogonal to both the laser and the string could in principle move the target ion outside the laser beam spot. To avoid this effect - and the consequent less efficiency - the laser beam can be focused by a cylindrical lens. This optical element can be arranged to produce a focal segment orthogonal to the directions of both the laser and the string. In this way the locations where the laser beams impinge on the string are two thin regions; these can be as wide as several hundreds of microns in one dimension without overlap between them. Transverse oscillations of a very cold ion beam are expected to be lower than this value. 


\section{AN APPLICATION OF THE METHOD TO A REAL STORAGE RING}

We shall discuss a possible implementation of such diagnostic device with reference to the case of $\mathrm{a}^{24} \mathrm{Mg}^{+}$string. In order to provide an example of a possible application, we shall refer to the ASTRID Storage Ring at Aarhus: its main parameters can be found in Ref. [12]. With the above assumptions, the velocity of the ion beam is about $v=8.97 \cdot 10^{5} \mathrm{~m} / \mathrm{s}$. The time duration of each laser pulse must be much shorter than the time, $T=s / v=56$ ps, taken by an ion to travel one interparticle spacing. On the other hand, the laser pulse must not be too short because this would lead to a very broad frequency pattern, in turn making more difficult the filtering between laser photons and fluorescence photons, as discussed below. A laser whose pulses are 2 ps long meets these requirements.

In the following we shall refer to a commercially available, frequency tripled $\mathrm{Ti}: \mathrm{Al}_{2} \mathrm{O}_{3}$ pulsed laser (50 MHz repetition rate), with some $\mathrm{nJ} /$ pulse. Considering the finite duration of a Gaussian-shaped laser pulse, the probability to excite a ${ }^{24} M g^{+}$ion is about 0.5 for the transition under consideration $\left(3 s^{2} S_{1 / 2} \rightarrow 3 p^{2} P_{1 / 2}\right)$. The laser frequency must be resonant with the ion's transition energy in its reference system $(\lambda=279.6 \mathrm{~nm})$. The laser beams need focusing to spot much smaller than the interparticle spacing $(50 \mu \mathrm{m})$. This can be done since it is experimentally possible to focus a laser beam within $5 \mu \mathrm{m}$ (FWHM). The laser focusing systems need to be placed in the vacuum chamber and should be movable in order to avoid interference with the ion beam during normal operation of the storage ring.

The deacay region is viewed by four photomultipliers, located each behind its own window. The window length $(2 L)$ matches the decay length for the ion de-excitation. The four windows cover about $50 \%$ of the azimuthal acceptance of the fluorescence photons. The distance $d$, between the upstream edge of photomultiplier acceptance and the interaction region between the ion and the laser beam should be as short as possible (see Fig. 2). We assume $d=10 \mathrm{~mm}, 2 L=20 \mathrm{~mm}$, a beam-pipe radius, $R=35 \mathrm{~mm}$, and the lifetime of the upper level, $\tau=3.5$ ns. The photomultipliers are single-photon detectors, which can be assumed to have a quantum efficiency of $23 \%$ and a rate of background counts of about 100 
c/s.

The signals from the four photomultipliers are discriminated (20 ns signal width) and the logical signals are ANDed two by two to form 6 combinations. These are then ORed; a positive logical level for the $\mathrm{OR}$ is an event in which the two ions, excited by the laser beams, have both emitted a photon.

Each photomultiplier must be equipped with a filter to intercept stray laser photons. Let $\theta$ be the angle between the direction of the ion beam and the direction of a photon emitted by an ion. Due to the finite duration of a laser pulse (2 ps), the frequency spectrum is of the order of $500 \mathrm{GHz}$. Considering that the laser beams impinge on the string at right angle, the photons of the lasers are overlapped in frequency with those from natural fluorescence between the angles $\theta=85^{\circ}$ and $\theta=95^{\circ}$. Filters are designed in order to discard all photons impinging with an angle $\theta>70^{\circ}$. The geometrical acceptance of the system allows detections of photons only for $\theta>50^{\circ}$. This corresponds to a lower limit for the filter bandwidth of $0.25 \mathrm{~nm}$. The distance between the two laser beam spots can be varied by moving the optical set-up; this movement can be done with an accuracy better than the range of longitudinal oscillation for interparticle spacing.

As described above, by moving the second laser beam with respect to the first one and counting the coincidence events of the photomultipliers, one can assess if the distance between the two laser-to-ion crossing points is an integer multiple of the string's interparticle spacing. After having met this condition, by slightly moving the second laser, one would record only accidental coincidences; this is true since, if the first laser excites an ion, then the second laser beam can no longer be synchronous with any other ion in the string. Appearance of correlation in the signals with the same periodicity of the string would be firm indication of ordering in the beam.

The total probability that one of the four detectors sees the fluorescence of an ion excited by one of the lasers is about $9.3 \cdot 10^{-4}$. Considering a purely random jitter of the laser, a laser beam spot of $5 \mu \mathrm{m}$ and a typical interparticle spacing of $50 \mu \mathrm{m}$, the probability that a laser pulse crosses an ion in the beam can be roughly estimated as $1 / 10$. 
Therefore, the probability that a simultaneous de-excitation will be recorded by the device is $1 \cdot 10^{-6}$. Considering a repetition rate of the laser of $5 \cdot 10^{7} \mathrm{~Hz}$, one expects a counting rate of approximately $50 \mathrm{~Hz}$. When the distance of the laser-to-ion beam crossing points is not an integer multiple of the string's step, the rate of accidental coincidences is estimated to be $0.25 \mathrm{~Hz}$. On the contrary, a non ordered ion beam would exhibit a coincidence rate of $10 \mathrm{~Hz}$ independent of the relative positions of the laser beams.

In order to check these results, we have developed a Monte Carlo simulation. Ion oscillations around their equilibrium positions, the excitation process of an ion by the laser light, the spontaneous emission, the geometrical acceptance and efficiency of the detector (filters + photomultipliers) are taken into account. Fig.3 shows the counting rate of coincidences versus the position of the second laser. A strong correlation signal is achieved for the case of an ordered string.

\section{CONCLUSIONS}

A novel method to detect ordered structures of an ion beam in an unambiguous way is proposed, and its feasibility is demonstrated. The method provides firm observation of ordering within the ion beam using available technology. This could be profitably applied to operating storage rings.

\section{ACKNOWLEDGMENTS}

The authors are grateful to A. Burov, R. W. Hasse, S. Gustafsson, L. Piemontese, and L. Tecchio for critical reading of the manuscript. 


\section{REFERENCES}

[1] F. Diedrich, E. Peik, M. Chen, W. Quint, and H. Walther, Phys. Rev. Lett. 59 (1987) 2931; D.J. Wineland, J.C. Bergquist, W.M. Itano, J.J. Bollinger, and C.H. Hanney, Phys. Rev. Lett. 59 (1987) 2935.

[2] I. Waki, S. Kassner, G. Birkl, and H. Walther, Phys. Rev. Lett. 68 (1992) 2007.

[3] See for example: Proc. of the International Workshop on "Light Induced Kinetic Effects on Atoms, Ions and Molecules", edited by L. Moi, S. Gozzini, C. Gabbanini, E. Arimondo, and F. Strumia (ETS, Pisa, 1991); Special issue on "The Physics of Trapped Ions", J. Mod. Opt 39 (1992) 193-443

[4] J.P. Schiffer, Z. Phys. A 321 (1985) 181.

[5] G.I. Budker, Atom. Energ. 22 (1967) 346.

[6] G. Bisoffi et al., "CRYSTAL, a Storage Ring for Crystalline Beams", LNL Int. Rep., INFN 50/94.

[7] R. Neumann, "Possible Laser Diagnostic Methods for Ordered Systems", Proc. of the "Workshop on Crystalline Ion Beams", Wertheim, October 1988, GSI-Report 89-10, p. 184.

[8] R.W. Hasse and J.P. Schiffer, Ann. Phys. 203 (1991) 419.

[9] L.D. Landau and E.M. Lifshitz, Statistical Physics (Pergamon Press, London, 1958), p. 482 .

[10] V.J. Emery and J.D. Axe, Phys. Rev. Lett. 40 (1978) 1507.

[11] V.V. Avilov and I. Hofmann, Phys. Rev. E 47 (1993) 2019.

[12] J.S. Nielsen, J.S. Hangst, O. Poulsen, J.P. Schiffer, P. Shi, and B. Wanner, Proc. of the "Workshop on Beam Cooling and Related Topics", Montreaux, October 1993, CERN 94-03, p. 339 and references therein. 


\section{FIGURES}

FIG. 1. A sketch of the experimental apparatus: a) general view, b) side-view.

FIG. 2. A sketch of the ion-laser interaction region.

FIG. 3. Counting-rate of coincidences versus second laser position for a string and for a hot beam with the same density . The acquisition time of events for the Monte Carlo is $1 \mathrm{~s}$. 


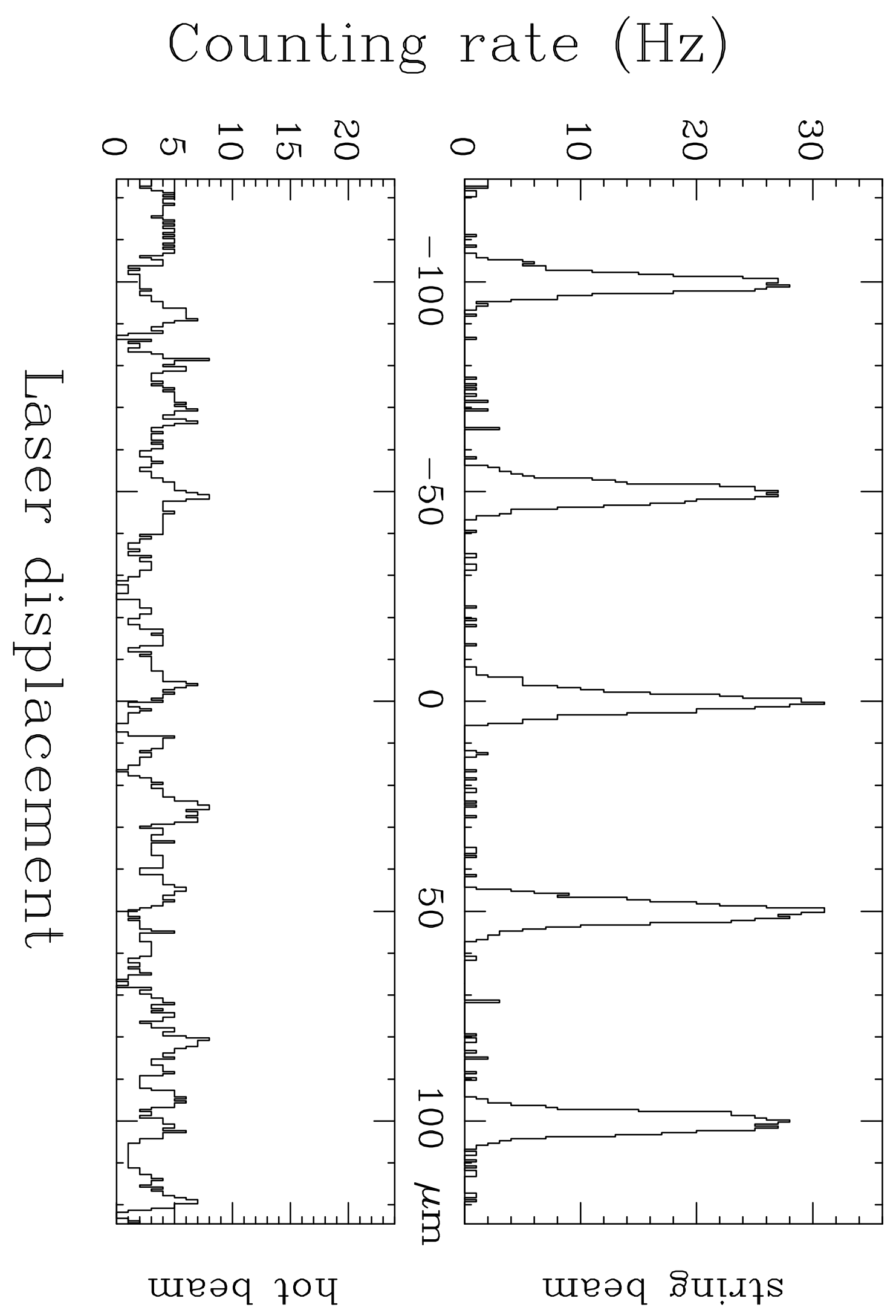

